Cipango Cahiers d'études japonaises

$18 \mid 2011$

Le Japon et le fait colonial I

\title{
Hokkaidō, an zéro
}

Changement des rapports de domination et septentrion japonais à la fin du XIX ${ }^{\mathrm{e}}$ siècle

\section{Noémi Godefroy}

\section{(2) OpenEdition}

Journals

\section{Édition électronique}

URL : https://journals.openedition.org/cipango/1517

DOI : 10.4000/cipango. 1517

ISSN : 2260-7706

Éditeur

INALCO

\section{Édition imprimée}

Date de publication : 1 juin 2011

Pagination : 105-133

ISBN : 978-2-85831-195-8

ISSN : 1164-5857

Référence électronique

Noémi Godefroy, « Hokkaidō, an zéro », Cipango [En ligne], 18 | 2011, mis en ligne le 17 juin 2013, consulté le 30 juin 2021. URL : http://journals.openedition.org/cipango/1517 ; DOI : https://doi.org/ 10.4000/cipango. 1517

Ce document a été généré automatiquement le 30 juin 2021

\section{(c) (†) \&)}

Cipango est mis à disposition selon les termes de la Licence Creative Commons Attribution - Pas d'Utilisation Commerciale 4.0 International. 


\section{Hokkaidō, an zéro}

Changement des rapports de domination et septentrion japonais à la fin du XIX ${ }^{\mathrm{e}}$ siècle

\section{Noémi Godefroy}

1 Avant d'être une colonie du Japon moderne, puis un élément à part entière de son territoire national, l'actuelle île de Hokkaidō, désignée avant 1869 par le nom d'Ezo, était déjà inscrite depuis longtemps dans un jeu complexe et changeant de rapports de domination à l'avantage de Honshū, l'île principale du Japon, et de ses habitants. Les Aïnous, les indigènes d'Ezo, étaient soumis à rude épreuve par leur proximité grandissante avec Honshū, et les habitants, puis colons de cette dernière découvraient à leur tour une « altérité » qu'ils allaient tendre à toujours dominer davantage.

2 Les troubles insurrectionnels des Aïnous, à partir du milieu $d u x^{e}$ siècle, alliés à l'importance stratégique de lî̂le, à la fin du $\mathrm{xvI}^{\mathrm{e}}$ siècle, dans l'optique d'envahir la Corée $^{1}$, finiront par rendre nécessaire un contrôle renforcé de la région. Un nouveau fief est donc créé dans la partie méridionale de l'île en 1590 : le fief de Matsumae (Matsumae han 松前藩). Ce dernier occupe une petite partie de la péninsule d'Oshima, et le clan Kakizaki 蟅崎 (qui deviendra le clan Matsumae, du nom de leur fief, en 1599) est placé à sa tête.

3 Par ailleurs, face aux nouvelles altérités politiques auxquelles l'archipel va se retrouver confronté à partir de la seconde moitié du xvI ${ }^{\mathrm{e}}$ siècle (Angleterre, Espagne, Portugal et Pays-Bas), les Tokugawa mettront en place, dans les années 1630, des interdictions maritimes (kaikin seisaku 海禁政策) qui «verrouillent» le pays en réduisant sévèrement ses échanges commerciaux et relations diplomatiques avec l'étranger. Une des conséquences de cette politique - qui sera qualifiée, plus tard, par les Européens de politique du "pays verrouillé » (sakoku 鎖国) -, est l'importance accrue des quelques relations commerciales encore autorisées, dont celles avec les Aïnous.

4 En effet, ces échanges permettent de contourner en partie les restrictions et de se procurer des produits continentaux (chinois, aïnous, nivkhs, toungouses ou russes). Ce commerce, dit de «santan » (santan kōeki 山丹交易) $)^{2}$, est extrêmement prospère. Le fief de Matsumae fournit au reste de l'archipel les fameux «trois produits d'Ezo » (Ezo no sanpin 蝦夷の三品) que sont le hareng (dont la chair broyée est utilisée comme 
engrais), le saumon, ainsi qu'une algue comestible, le konbu 昆布 (famille des Laminariaceae). S'ajoutent à cela les plumes de faucon et l'emblématique «brocart d'Ezo » (Ezo nishiki 蝦夷錦). Comme la riziculture n'est pas pratiquée avant les années 1850 à Ezo, l'une des particularités du fief de Matsumae est donc qu'il ne participe pas au système de kokudaka ${ }^{3}$. Les familles de guerriers dépendant du fief ne reçoivent pas de terres, comme c'est le cas dans le reste de l'archipel, mais des droits de commerce exclusifs avec les Aïnous. Ce commerce est limité à des lieux prédéfinis par le fief et octroyés aux vassaux les plus fidèles, ce qui permet d'exercer un contrôle indirect sur ce commerce bilatéral. Ce « système des comptoirs de commerce alloués " (akinaiba chigyō sei 商場知行制) voit le jour en 1596. De ce fait, un système de dépendances croisées se met en place, et dans son sillon, des relations coercitives : les Aïnous sont dépendants du fief pour se procurer des produits «japonais», le fief utilise les Aïnous pour justifier sa position de plaque tournante commerciale et son statut particulier vis-à-vis du shogunat ${ }^{4}$, ainsi que pour remplir les caisses domaniales en l'absence de riziculture. Les Aïnous, dans le cadre de cette relation tributaire, sont donc à la fois fournisseurs, marché et main-d'œuvre.

5 La politique économique du fief est donc intrinsèquement liée à sa politique indigène ; sa position dominante doit être sans cesse réaffirmée vis-à-vis des Aïnous, comme visà-vis du shogunat. Dans cette optique, l'altérité des indigènes est mise en exergue. Une manifestation concrète en est la cérémonie de remise des tributs (uimamu ウイマム) organisée tous les ans à Matsumae. Les Aïnous, en costume d'apparat, sont paradés dans toute la ville avant d'être reçus par le seigneur. Leur apparence exotique est mise en scène et sert de justification à la domination. Des cérémonies de plus petite envergure, mais plus fréquentes - les omusha オムシャ -, sont organisées autour des comptoirs commerciaux, en territoire aïnou. ${ }^{5}$ En outre, cette domination passe également par le maintien de ces populations dominées dans une relative ignorance: durant presque toute la période de domination du fief, il leur est ainsi interdit d'apprendre la langue et les coutumes de leurs maîtres. Dès le début du XVIII ${ }^{\mathrm{e}}$ siècle, les comptoirs de commerce susnommés se muent en "lieux d'entreprise contractuels » (basho ukeoi 場所請負). Pour faire face à une demande commerciale grandissante, ces basho, gérés par des vassaux du fief, puis par des commerçants, servent à la fois de lieux d'échanges, mais également d'exploitation de la main-d'œuvre aïnoue, dans ce que l'on pourrait qualifier de pêcheries proto-industrielles ${ }^{6}$.

\section{La nécessité grandissante d'un nouveau type de domination}

6 L'Empire russe fait preuve d'un intérêt croissant pour les marges orientales de son territoire et, à la fin du XVII e siècle, des garnisons sont installées en Yakoutie, dans le nord-est de la Sibérie. En 1711, les Cosaques Danila Antsiferov et Ivan Kozyrevski entreprennent l'exploration des Kouriles (Antsiferov y est d'ailleurs tué, en 1712, par ses indigènes), et de petites colonies russes commencent à s'y installer. C'est grâce, entre autres, à la présence de naufragés japonais que la Russie de Pierre le Grand prend conscience de sa toute nouvelle proximité géographique avec l'archipel japonais? ${ }^{7}$.

7 Les Russes entrent en contact avec les Japonais présents sur l'île d'Ezo dès 1778, mais ces derniers refusent catégoriquement tout commerce avec eux, en vertu des interdictions maritimes. Cette demande est renouvelée en 1792, et refusée de nouveau. 
Par ailleurs, de plus en plus de navires occidentaux sillonnent la région en cette fin de XVIII ${ }^{\mathrm{e}}$ siècle $^{8}$.

8 Si la domination économique japonaise à Ezo ne fait alors aucun doute, la domination politique est encore faible, et la présence grandissante d'Occidentaux dans cette région du monde, ajoutée aux difficultés financières du shogunat, motive certains lettrés confucéens proches du shōgun à remettre en question la nature et les objectifs de la domination septentrionale.

9 À partir des années 1780, on voit apparaître dans l'entourage du shogun des « traités en faveur du défrichement» (kaitakuron 開拓論). Deux courants de pensée s'affrontent. Les partisans du statu quo, fidèles aux interdictions maritimes, veulent conserver l'île d'Ezo en tant que zone tampon non défrichée. D'après eux, un tel territoire serait d'autant moins un objet de convoitise territoriale qu'il n'est pas développé ${ }^{9}$.

10 À eux s'opposent ceux qui militent pour l'ouverture et le développement, voire une plus grande intégration de l'île au territoire sous domination directe des Tokugawa, s'inspirant des politiques territoriale, économique et militaire russes ${ }^{10}$. Ces derniers remettent par la même occasion en question le paradigme des interdictions maritimes et de la politique dite du sakoku, et ce bien avant l'arrivée de Matthew Perry.

11 Ce qui est innovant, c'est qu'aux arguments économiques des partisans du changement s'ajoutent des arguments stratégiques. Cependant, comme le souligne Augustin Berque $^{11}$, ils ont tous en commun de raisonner d'après l'état de la zone dominée par le shogunat, et non d'après la situation de l'ensemble de l'île : une écrasante majorité de ces lettrés ne s'y est d'ailleurs jamais rendue. En fin de compte, c'est l'évolution du contexte international qui va donner un poids décisif aux thèses de ces derniers.

En effet, à la fin du xvIII ${ }^{\mathrm{e}}$ siècle, la présence grandissante des Russes et de navires étrangers dans la région, ajoutés à une insurrection aïnoue dans les Kouriles en $1789^{12}$, sont à l'origine du passage de l'île d'Ezo sous administration shogunale directe à partir de 1799. Si l'île revient sous le contrôle du fief entre 1821 et 1854 , un premier pas important vers l'intégration vient d'être franchi et ce sont désormais les orientations stratégiques face aux pressions extérieures, et non plus uniquement les objectifs "économiques» d'une relation tributaire, qui dictent dorénavant la nature de la domination sur l'île.

13 Après avoir analysé les rapports entre l'île d'Ezo et le Japon jusqu'à Meiji, on peut donc évoquer des relations de "colonialisme interne $»^{13}$, qui se rapprochent du concept de "sociétés supplantantes» (supplanting societies), défini par David Day ${ }^{14}$, ou d'un « rapport inégal de nature colonisatrice »". L'annexion de l'île d'Ezo au nouvel espace national en tant qu'île de Hokkaidō en 1869 parachève donc une dynamique de domination ancienne. Mais elle marque également une rupture nette avec la colonisation d'Ancien Régime: l'annexion de Hokkaidō au Japon est stratégique, consolidée par une orientation politique de défrichement, de peuplement, et d'intégration de la population aïnoue. Le Japon confirme ainsi qu'il se situe dans un paradigme de relations internationales nouveau : celui des États-nations.

\section{La naissance de Hokkaidō}

Après l'arrivée de Perry et l'abrogation, en 1854, des interdictions maritimes qui avaient en partie isolé l'archipel, le statut des marges septentrionales fait l'objet de 
nouvelles tractations entre puissances. Hakodate, l'un des deux ports ouverts aux Américains d'après le Traité de Kanagawa ${ }^{16}$, se trouve sur l'île d'Ezo. Mais, comme nous l'avons évoqué plus haut, c'est surtout pour la Russie que cette zone présente un intérêt particulier.

Le 7 février 1855, la Russie et le Japon signent le traité de Shimoda (ou Traité d'amitié russo-japonais, Nichiro washin jōyaku 日露和親条約), similaire au traité de Kanagawa. Une question délicate y est abordée : celle du problème de définition des frontières autour des Kouriles. La mainmise du nouvel État japonais sur Ezo est assurée, mais un partage entre les deux puissances est préconisé pour les territoires encore plus au nord : toutes les îles au sud de l'île d'Etorofu (actuelle Itouroup) seront japonaises, et toutes celles au nord d'Ouroup appartiendront à la Russie ${ }^{17}$.

En 1867, les deux États signent le Règlement provisoire concernant Karafuto [Sakhaline] et Chishima [les Kouriles] (Karafuto Chishima ni kan suru karikisoku 樺太千島二関スル仮規 則), mais celui-ci ne règle en rien le problème de l'île de Sakhaline, qui est provisoirement reconnue comme une "zone de peuplement mixte russo-japonais " (zakkyochi 雑居地), c'est-à-dire un condominium ${ }^{18}$. Les tensions russo-japonaises, toujours très vives malgré la signature de traités, sont déterminantes dans le choix de l'empereur Meiji d'annexer officiellement l'île après 1868.

\section{L'annexion de l'île d'Ezo}

La fonction de l'île d'Ezo est de servir de porte nord à l'empire ; elle est en contact direct avec Santan et la Mandchourie. Les frontières sont grossièrement établies, et cette partie nord est occupée par plusieurs peuples [japonais, aïnou et russe]. Jusqu'à maintenant, les administrateurs [de l'île] se sont servis des indigènes de manière extrêmement cruelle alors que les étrangers [les Russes] se montraient très compatissants. Les indigènes éprouvent donc souvent de la rancœur contre nous tandis qu'ils leur vouent respect et confiance. Quand, prétextant d'aider ce peuple face à ses difficultés, il se trouvera des gens pour agiter ces indigènes, cette agitation calamiteuse s'étendra comme une trainée de poudre à Hakodate et Matsumae. Pour prévenir ceci, notre travail à présent, une fois Hakodate pacifiée est la mise en œuvre immédiate de moyens pour guider la politique de défrichement, et faire [de l'île d'Ezo] une terre où le peuple pourra croitre. 蝦夷地之儀八皇国ノ北門、直二山丹 ・満州二接シ、経界粗定卜イへ共、北部二 至テハ、中外雑居致候処、是迄官使之土人 八頗ル愛恤习施シ候ヨリ、土人往々我邦人ヨ怨離シ、彼习尊信スルニ至ル。一 但民苦ヨ救フヨ名トシ、土人ヨ脲動スル者有之時八、其禍忽于箱館、松前二延 及スルハ必然二テ、禍ヨ未然二防ク八、方今ノ要務二候間、箱館平定ノ上八、 速二開拓教導等之方法ヨ施設シ、人民繁殖ノ城トナサシメラルベキ儀二付、利 害得失、各意見無忌憚可申出候事 ${ }^{19}$ 。

17 C'est avec ces mots que l'empereur Meiji fixe, dans une allocution à son cabinet le 30 juin 1869 , une nouvelle priorité gouvernementale au régime : le défrichement et le peuplement des marges septentrionales de l'empire. Cette décision fait suite à une déclaration précoce du ministère des Affaires suprêmes (Dajōkan 太政官), le 12 mars 1868, faisant du défrichement et du peuplement du territoire d'Ezo des orientations prioritaires de l'État.

En cette fin de XIX $x^{e}$ siècle, dans un contexte de pressions occidentales grandissantes sur le plan extérieur et de troubles accompagnant la restauration de la monarchie sur le plan intérieur - le gouvernement livre alors bataille contre les troupes pro-shogunales 
à Hakodate ${ }^{20}$ - une des priorités de l'État est la sécurisation de la frontière nord d'un territoire japonais se voulant unifié. C'est dans cette optique que le Japon annexe Ezo, le 20 septembre 1869, qui cesse officiellement d'exister et devient l'île de Hokkaidōo ${ }^{21}$. Cette annexion marque une rupture dans la nature, les objectifs et les moyens de la domination japonaise sur l'île. Les moyens mis en œuvre sont le défrichement du territoire, son peuplement, ainsi que l'intégration de la population indigène. Si Hokkaidō ne constitue évidemment pas le laboratoire de toutes les politiques coloniales menées par le Japon en Asie, son annexion possède toutefois un caractère pionnier dont l'analyse peut s'avérer utile à la compréhension de la politique extérieure japonaise qui suivra la Restauration de Meiji.

Une instance spécifique est créée pour piloter les opérations: la Mission au Défrichement (Kaitakushi 開拓使). Cette nouvelle instance gouvernementale et administrative est chargée de mettre en place l'intégration administrative du territoire et de sa population, ainsi que les premières mesures de défrichement. Elle guide la politique gouvernementale à Hokkaidō entre 1869 et 1882, mais les mesures qu'elle met en place - l'An Zéro pour Hokkaidō - se concentrent sur les années 1869-1872, c'est-àdire les deux premières années d'existence de la Mission et la première année de son Plan décennal (Kaitakushi jūnen keikaku 開拓使十年計画). On peut noter que si certaines décisions se basent sur les acquis de l'expérience de domination tributaire, d'autres, au contraire, partent sur des bases nouvelles et innovantes relevant de l'arsenal le plus moderne de la colonisation. La Mission au Défrichement fait donc office de pivot entre deux types de logiques.

\section{La mise en place de la Mission au défrichement}

La Mission voit le jour dans la capitale, récemment rebaptisée Tōkyō, le 2 juillet 1869, avec Matsuura Takeshirō 松浦武四郎22 comme responsable. Le gouvernement japonais lui confère les pleins pouvoirs, mais ne lui fournit pratiquement aucun fonds ${ }^{23}$. Nous y reviendrons.

21 En septembre, un bureau régional de la Mission voit le jour à Hakodate, qui constitue à l'époque le centre de gravité de l'implantation japonaise sur l'île, et dès le mois suivant, des antennes provisoires (kariyakusho 仅役所) sont mises en place à Otaru (octobre 1869-mai 1871), Nemuro (octobre 1869-juin 1870), Sōya (octobre 1869janvier 1870) et Sakhaline (d'octobre 1869 à février 1870) ${ }^{24}$. Leur mise en place accompagne les déplacements des responsables de la Mission qui parcourent l'île entre octobre 1869 et mai 1871 en vue d'organiser le défrichement à l'échelle régionale et locale. C'est n'est donc qu'en mai 1871 qu'est installé le bureau définitif de la Mission à Hokkaidō, sur le site de Sapporo de fondation récente ${ }^{25}$.

Cette relocalisation du pouvoir nous semble représentative d'une volonté politique de rompre avec l'organisation d'Ancien Régime du fief de Matsumae, en se rapprochant du centre géographique effectif de lîle. C'est de Sapporo que la Mission prend les toutes premières mesures d'appropriation de l'île, à commencer par celle du changement des toponymes, et tout d'abord celui qui désigne l'île même. 


\section{La Mission baptise l'île}

Après l'intégration de l'île au Japon, l'ancienne désignation "Ezo" devient une évocation constante de ses habitants d'origine ${ }^{26}$, de leur langue et de leur culture propres, ainsi que d'un passé distinct de l'île, lequel pourrait remettre en cause la légitimité de l'annexion japonaise. Rebaptiser le territoire selon une nouvelle désignation administrative prend de ce fait une importance toute particulière, inséparable du processus d'intégration moderne du Japon.

les premiers travaux de Matsuura, on sait déjà que l'ensemble des territoires septentrionaux sera enregistré sous le terme administratif de $d \bar{o}^{27}$. Le géographe rédige à l'intention du gouvernement un avis présentant six propositions de nom, la « Lettre concernant le choix définitif du nom des districts (gun), des régions (koku) et du dō de Hokkaidō» (Hokkaidōdō kokugunmei sentei jōsho 北海道々国郡名撰定上書) ${ }^{28}$ : Hidakamidō 日高見道 (la « province d'où l'on voit le soleil briller haut »); Hokka-idō 北加伊道 (la «province qui nous rejoint par le nord », mais «ka-i » renverrait à l'un des mots anciennement utilisés pour désigner les Ezo ${ }^{29}$ ); Kaihokudō 海北道 (la «province du nord de la mer ») ; Kaitōdō 海東道 (la «province de l'est de la mer »); Tōhokudō 東北 道 (la «province du Nord-Est»); et Chishimadō 千島道 (la " province de Sakhaline »). Pour chacune de ces propositions, il fournit des arguments justifiant son choix. La plupart sont d'ordre géographique, exception faite de Hidakamidō et Hokka.idō.

Hidakamidō est d'inspiration plus «classique». En effet, ce nom est inspiré d'un paragraphe des Annales du Japon (Nihon shoki 日本書紀) relatif à Takenouchi Sukune 武 内宿禰 ${ }^{30}$, qui évoque l'existence du «pays de Hidakami», dans les terres barbares ${ }^{31}$.

Finalement, c'est un mélange entre Hokka.idō et Kaihokudō qui est choisi : Hokkaidō 北 海道 (la «province de la mer du nord») reprend la prononciation du premier et les idéogrammes du second ${ }^{32}$. Par ailleurs, c'est un « clin d'œil » à l'un des pseudonymes de Matsuura : Hokkaidō-jin 北海道人 (homme de la province de la mer du nord).

\section{Une administration multicéphale}

Il faut désormais procéder à l'organisation du défrichement, qui nécessite à la fois le peuplement de l'île et une participation financière des différents fiefs. En effet, le budget total de la Mission ne dépasse pas les deux cent mille à deux cent cinquante mille yens entre 1869 et 1871 pour des dépenses annuelles estimées à huit cent soixante-dix mille yens. À partir de 1871 et de la mise en place du Plan décennal, le budget augmentera pour atteindre dix millions de yens sur dix ans. Ce budget décennal est équivalent aux dépenses totales de l'État japonais pour la seule année $1871^{33}$. On peut noter que malgré l'importance de l'intégration et du défrichement de Hokkaidō, le budget national ne semble pas suivre.

Dans cette optique, le 22 juillet 1869 , le ministère aux Affaires suprêmes proclame, par l'ordonnance 660 :

Selon la proclamation de l'empereur concernant le défrichement du territoire d'Ezo, les terres propres [à la culture] seront désormais cédées à tous ceux qui en feront la demande des guerriers jusqu'aux gens du peuple, pour qu'ils procèdent au défrichement.

蝦夷地開拓のことは、先般の御下問にもあった通り、今後諸藩士族庶民に至る まで、志願次第を申し出た者に相応の地を割譲し、開拓を仰せ付ける ${ }^{34}$ 。 
Suite à cette proclamation, seuls six fiefs et une famille se portent volontaires. Le gouvernement décide donc d'obliger les grands fiefs à participer à l'effort de défrichement. C'est ce qui est fait le 22 août de la même année, par la proclamation suivante ${ }^{35}$ :

Concernant l'exploitation de Hokkaidō, comme il a été ordonné précédemment, et selon la situation de devoir urgent dans laquelle nous nous trouvons, petit à petit, il faut mettre la main à la pâte ; le succès d'une telle entreprise est incertain à moins que tout le pays n'unisse ses forces, nous exigeons leur soutien aux fiefs listés en annexe avec les territoires qui leur sont confiés et nous leur demandons d'en effectuer une gestion assidue et efficace.

北海道開拓之儀八、兼厅被仰出候通即今之急務二テ、追々御手习被為著候 処、何分全国之力ヨ用ヒスン八成功無覚束、依之今般別紙地所其藩へ支配被仰 付候間、拮据経営実効相立候様可致事。

Cet ordre s'étendra peu à peu aux fiefs plus petits, puis à certaines familles de guerriers, à l'instar de celle de l'ancien fief de Sendai.

Hokkaidō est alors divisé en trente-huit zones (Hokkaidō no bunryō shihai 北海道の分領 支配). L'une d'elles est attribuée au ministère des Armées (Hyōbushō 兵部省), une autre au gouvernement métropolitain de Tōkyō (Tōkyō-fu 東京府), deux à des maisons de la noblesse de cour, deux à des temples, huit à des maisons de l'aristocratie guerrière et vingt-quatre à des fiefs. Ceux-ci doivent prendre en charge les frais de son exploitation et se charger de l'envoi de colons ${ }^{36}$. La Mission, quant à elle, sert d'instance unificatrice (tōkatsu kikan 統括機関); elle dicte l'orientation générale du défrichement, en fixe les objectifs chiffrés et représente l'autorité judiciaire sur place (Cf. tableau suivant.)

Seuls treize fiefs, six familles de l'aristocratie guerrière et deux institutions bouddhiques prennent en charge leur zone jusqu'à la fin de vie de cette mesure, lors de l'abolition des fiefs et la création des départements le 29 août 1871 (haihan chiken 廃藩 置県).

\section{Le peuplement de l'île}

Il est crucial pour la Mission au défrichement de peupler Hokkaidō le plus rapidement possible et ce pour plusieurs raisons. La première est que le défrichement et la mise en valeur de l'île nécessitent une main-d'œuvre importante et les quelque dix-sept mille Aïnous et soixante-mille Japonais déjà présents sur place $^{37}$ ne suffisent pas. De plus, une colonisation de peuplement légitime de fait l'annexion, tout en dissuadant d'éventuelles velléités russes dans la région (hokuhen bōbi 北辺防備). Par ailleurs, l'envoi sur l'île de familles de l'ancienne aristocratie guerrière, classe qui vient d'être abolie, participe à leur «reclassement » (shizoku jusan 士族授産). Désormais privés de leur pension, ceux-ci se retrouvent sans emploi et en grande difficulté financière au début de l'ère Meiji.

Attribution des trente-huit zones du territoire de Hokkaidōo (septembre 1869)

\begin{tabular}{|c|c|c|c|c|}
\hline $\begin{array}{l}\text { Fiefs } \\
\text { volontaires } \\
(15)\end{array}$ & $\begin{array}{l}\text { Fiefs non } \\
\text { volontaires } \\
(9)\end{array}$ & $\begin{array}{l}\text { Familles nobles } \\
(2)\end{array}$ & $\begin{array}{l}\text { Familles de } \\
\text { guerriers } \\
(8)\end{array}$ & $\begin{array}{l}\text { Autres } \\
\text { (5) }\end{array}$ \\
\hline
\end{tabular}




\begin{tabular}{|c|c|c|c|c|}
\hline Saga* & Fukuoka* & Tayasu (Yoshiyori) & Date (Kunishige)* & Zōjōji \\
\hline Tokushima & Kagoshima & $\begin{array}{l}\text { Hitotsubashi } \\
\text { (Mochiharu) }\end{array}$ & Date (Kuninao)* & Bukkōji \\
\hline Kōchi* & Kumamoto & & Date (Hirotaka)* & \\
\hline Mito* & Yamaguchi* & & $\begin{array}{l}\text { Ishikawa } \\
\text { (Kunimitsu) }\end{array}$ & $\begin{array}{l}\text { Ministère } \\
\text { des } \quad \text { Affaires } \\
\text { militaires }\end{array}$ \\
\hline Ichinoseki* & Hiroshima & & $\begin{array}{l}\text { Katakura } \\
\text { (Kuninori)* }\end{array}$ & \\
\hline Ōizumi & Wakayama & & $\begin{array}{l}\text { Watari } \\
\text { (Tanemoto)* }\end{array}$ & $\begin{array}{l}\text { Gouvernement à } \\
\text { Tōkyō }\end{array}$ \\
\hline Akita* & Nagoya & & $\begin{array}{l}\text { Gotō } \\
\text { (Sanenosuke) }\end{array}$ & \\
\hline Hirosaki* & Kanazawa & & Inada (Kunitane)* & $\begin{array}{ll}\text { Mission } & \text { au } \\
\text { Défrichement } & \end{array}$ \\
\hline Tonami* & Shizuoka* & & & \\
\hline Yonezawa* & & & & \\
\hline Tottori & & & & \\
\hline Okayama & & & & \\
\hline Fukuyama & & & & \\
\hline Hikone* & & & & \\
\hline Sendai* & & & & \\
\hline
\end{tabular}

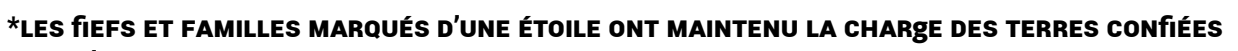
JUSQU'EN 1871

En octobre 1869, une première mesure réglementant la colonisation voit le jour: le 《Règlement pour l'aide au peuplement 》 (Imin fujo kisoku 移民扶助 規則). Celle-ci divise les colons en quatre catégories : les paysans et ouvriers agricoles sous contrat, les commerçants et artisans sous contrat (Bōi nōfu u 募移農夫, Bōi shōkō 募移商工), et leurs pairs venus d'eux-mêmes (Jii nōfū 自移農夫, Jii shōkō 自移商工). Leur sont fournis trois mois de vivres (principalement du riz et du sel), des outils, des graines, et de quoi se loger, en plus de six mille $t_{s u b o^{38}}$ (l'équivalent d'environ deux hectares) de terrain à défricher. Cette mesure est prise dans les territoires sous contrôle de la Mission au défrichement, mais pas dans les trente-huit autres zones. Elle reste donc assez limitée, mais à partir de 1871, année de la mise en place du Plan décennal, les mesures agraires prendront, comme nous le verrons, de l'ampleur. 
Malgré cette première mesure, d'après une étude sur les migrations internes qui analyse le cas de Hokkaidō entre 1869 et $1925^{39}$, il n'y a pas de pics de migration à Hokkaidō avant la mise en place des mesures terriennes dans le cadre du Plan décennal. L'immigration à Hokkaidō entre 1869 et 1872 est donc assez sporadique et très localisée; les vagues successives d'immigration de masse débuteront quant à elles en $1891^{40}$. Ceux qui forment le plus gros contingent entre 1869 et 1872 sont les familles de guerriers. C'est le cas, entre bien d'autres, de la Maison Date, hostile à la restauration de Meiji, qui doit quitter le district de Watari, dans le fief de Sendai, à la suite de son chef Date Kunishige 伊達邦成. Elle se rend dans le district d'Usu dans la région d'Iburi ${ }^{41}$, et $y$ fonde une ville qui porte son nom en 1870 . Entre 1870 et 1881 , cinq cent soixante-dix foyers de guerriers du fief de Sendai s'installent à Hokkaidō.

Certains écrivains japonais ont pu idéaliser l'arrivée de ces premiers migrants et décrivent de manière dithyrambique leur esprit aventurier, à l'instar de Kamei Katsuichirō 亀井勝一郎, dans la décennie précédant le centenaire de l'annexion de Hokkaidō.

Il ne fait aucun doute qu'un esprit d'aventure habitait les colons des premiers temps, l'aventure de " construire un pays », un nouveau pays sur cette terre vierge. [...] Peut-être y avait-il une dimension fantasmagorique, une sorte d'impulsion quasi-artistique, dans l'idée de construire un nouveau pays, en défrichant des forêts primaires et des étendues herbeuses. [...] Dans un processus artistique, il n'y a pas de rêve plus grand que la « construction d'un nouveau pays».

この処女地媇しい国を建てようといふ云はば『国づくり』の冒険心が初期の

移住民にあったのは事実である。（略）広漠とした原野や原始林をきりひらい

て、新しい国をつくらうといふ行為の中で、『国づくり』ほど大規模で夢ふか

いものはあるまい42。

37 Ces propos ne différencient pas les preneurs de décision (à Sapporo) et les exécutants (les migrants à l'intérieur de l'île), faisant presque croire à une sorte d'élan spontané, naturel à l'Homme, dénué de toute intention politique ou considération économique. En fait, si l'on exclut les groupuscules de guerriers qui rêvaient de réinstaurer à Hokkaidō l'ancien régime shogunal, il n'est pas clair qu'il eût à un quelconque moment une idéologie sous-jacente de "construction d'un nouveau pays» chez les leaders intermédiaires, ou parmi les masses de migrants forcés au départ par des conditions de vie intenables et qui avaient perdu leurs moyens de subsistance à l'application d'un nouveau principe : celui de la propriété privée. Pour reprendre les termes de l'historien Kaiho Mineo, «Hokkaidō était un endroit où migraient ceux qui n'avaient plus nulle part où fuir »(逃げ場のなくなった民衆) ${ }^{43}$.

Le film Kita no zero nen 北の零年 (Le Nord: An 0) $)^{44}$ - dont est tiré le titre de cette contribution - dépeint lui aussi de manière très romancée les premières années très difficiles de la vie de ces colons. Il dépeint le combat quotidien de la famille Inada du fief de Tokushima contre les rudesses du climat, la faim et la maladie, l'aide inattendue qu'ils reçoivent des Aïnous et le profond sentiment d'abandon qu'ils ressentent de la part des autorités locales. Cette histoire n'est pas sans rappeler le mythe fondateur du repas de Thanksgiving ${ }^{45}$, aux États-Unis. C'est d'ailleurs en grande partie sur l'expérience américaine que la Mission va d'ailleurs s'appuyer pour effectuer le découpage, défrichement et peuplement de Hokkaidō. 


\section{«Boys, be ambitious ! » : l'exemple colonial américain}

Comme nombre d'institutions créées par le nouveau régime politique japonais, la Mission au défrichement ressent le besoin de faire appel à des experts occidentaux pour acquérir les techniques nécessaires à la réussite de ses objectifs : redistribuer les terres aux migrants japonais et défricher le territoire en mobilisant conjointement colons et population locale. Elle porte son choix sur les États-Unis et on peut y voir plusieurs raisons. En effet, de par la progression du front de la conquête de l'Ouest, le gouvernement américain a affaire à des problématiques similaires (peuplement, redistribution terrienne, défrichement, gestion de la question indienne). De plus, les États-Unis prônent une logique capitaliste de surplus, que le Japon souhaite développer à Hokkaidō. On peut encore invoquer des conditions climatiques similaires dans certains états, l'avancée technologique américaine en matière de matériel agricole, ainsi que peut-être une perception également inquiète chez les États-Unis de l'avancée russe vers le Pacifique et l'Alaska ${ }^{46}$.

En 1871, le sous-directeur de la Mission au défrichement, Kuroda Kiyotaka 黒田清隆 ${ }^{47}$, fait venir une délégation de conseillers américains, dont Horace Capron ${ }^{48}$.

Celui-ci avait servi de commissaire à l'Agriculture sous les présidents Andrew Johnson et Ulysses S. Grant. Au Japon en tant que « spécialiste étranger invité » (oyatoi gaikokujin お雇い外国人), Capron participe à la rédaction des premières mesures terriennes, et contribue à l'établissement de routes, de mines, d'exploitations agricoles et d'élevages de grande envergure, ainsi que d'usines. Hokkaidō lui doit la construction de la ville de Sapporo selon le plan en damier des grandes villes américaines. Il préconise par ailleurs la culture de l'orge dans lîle, rendue possible par le climat, ce qui mènera indirectement à la fabrication de la première bière japonaise à Sapporo. Il quitte le Japon en 1875, non sans avoir préconisé pour la gestion de la question aïnoue les mêmes mesures que celles utilisées à l'encontre des nations indiennes: la dispersion des communautés pour diminuer le risque de poches de résistance, la mise en place d'un système de soldats-paysans, pour surveiller les indigènes, mais aussi les colons ${ }^{49}$.

\section{Les premières mesures agraires}

L'année 1871 marque un tournant dans le développement de Hokkaidō. Le 29 août, on assiste à l'abolition du système de fiefs et avec elle, la fin de leur soutien financier dans l'effort de défrichement de Hokkaidō. De ce fait, la Mission reprend le plein contrôle direct et effectif de l'île et pose les bases de son Plan décennal, qui est mis en place l'année suivante. Ce plan permet la mise en place des premières mesures agraires en 1872. Celles-ci sont au nombre de deux : le Règlement sur la terre à Hokkaidō (Hokkaidō jisho kisoku 北海道地所規則) et le Règlement sur la vente et la location de terrains à Hokkaidō (Hokkaidō tochi urikashi kisoku 北海道土地売貸規則). Elles sont confirmées l'année suivante par le Décret révisé sur la régulation foncière (Chiso kaisei jōrei 地租改正条 例 $)^{50}$.

La première affirme que le territoire de Hokkaidō est considéré par le gouvernement japonais comme étant terra nullius, « une terre sans maître» (無主の地 mushu no chi). Par conséquent, à l'exception des «terres au cœur des montagnes et dans des vallées profondes, vierges de toute trace humaine»(深山幽谷・ 人跡隔絶 Shinsan yūkoku 
jinseki kakuzetsu), tous les terrains sont libres de devenir propriété privée japonaise. La vente et la location de ces terres sans propriétaire constituent une double aubaine pour le gouvernement japonais et la Mission au défrichement : générant des bénéfices nets sur des terres obtenues gratuitement, elles attirent également colons et sociétés exploitantes, main-d'œuvre et capitaux.

La seconde mesure, le Règlement sur la vente et la location de terrains à Hokkaidō, a pour but d'attirer les acheteurs potentiels japonais en leur proposant des conditions de vente très avantageuses, et de faire venir ainsi des colons motivés par l'exploitation des terres. Grâce à ce règlement, chaque acheteur peut acquérir jusqu'à cent mille tsubo (environ trente-trois hectares) par personne, exempts de taxes pendant dix ans. La présence de spécialistes invités américains est ici intéressante : la mesure n'est pas sans rappeler le Homestead Act de 1862, qui permettait d'acquérir pour une très petite somme quatre-vingts hectares de terrain dans les territoires de l'Ouest américain après y avoir vécu (et donc les avoir défrichés) pendant au moins six mois ${ }^{51}$.

Ces règlements sur la terre et sur la vente sont mis en application rapidement, et sans concertation avec les populations locales, qui vivent pourtant sur ces terres fertiles, vendues au plus offrant. Elles sont à l'origine du premier pic d'immigration japonaise à Hokkaidō et attirent environ treize mille colons entre 1872 et $1873^{52}$. Ces mesures agraires ne sont que les premières d'une longue série, qui verra les Aïnous être déplacés de force à partir de 1875, par les soldats-paysans, dans le but de libérer les terres pour les distribuer aux colons.

\section{La gestion de la question indigène}

\section{L'abolition des basho}

L'objectif prioritaire de la Mission vis-à-vis des Aïnous est leur mobilisation comme main-d'œuvre de l'effort de défrichement. Pour cela, il faut les libérer du système des «lieux d'entreprise contractuels", dont le joug pèse sur eux depuis le XvIII siècle. Comme on l'a vu plus haut, les basho, devenus en majorité des exploitations piscicoles ou de déboisement, emploient des Aïnous en tant que travailleurs précaires, voire forcés ${ }^{53}$. Ce système, dénoncé par de nombreux administrateurs, tel Matsuura Takeshirō, comme étant cruel et corrompu, représente en 1869 un obstacle à la politique de défrichement, pour deux raisons : il entrave la redistribution des terres, occupées par les basho, aux colons japonais prévue par la Mission au défrichement; il empêche que les Aïnous qui y sont employés puissent servir de main-d'œuvre au défrichement de l'île. La Mission se heurte ainsi frontalement à l'ancienne organisation mise en place durant l'Ancien Régime japonais, qui représente pour elle une gêne dans ses nouveaux objectifs. Ils sont abolis le 28 septembre 1869.

Cette abolition ne transforme toutefois pas radicalement les rapports de domination sur l'île. Une majorité des entrepreneurs en charge des basho, riches commerçants et industriels, parviennent à maintenir toute leur influence dans la région grâce à d'importants pots-de-vin versés aux administrateurs de la Mission au défrichement ${ }^{54}$. Celle-ci est donc doublement bénéficiaire dans cette opération : elle est débarrassée du système contraignant des basho et a désormais le champ libre pour exploiter les Aïnous à sa guise. 

souvent constatée lors de l'abolition d'un système servile: ils se retrouvent certes libres, mais sans emploi et sans protection minimale, main-d'œuvre abondante, peu coûteuse et docile.

\section{L'assimilation administrative des Aïnous}

Tout comme l'attribution d'un nom nouveau permettait l'annexion du territoire, il fallait attribuer un statut nouveau aux Aïnous, qui autoriserait leur intégration. En 1868, «Ezo » rappelle l'existence d'un territoire resté non japonais jusqu'à Meiji. Quant au terme " Aïnou » (« être humain », en langue aïnoue), il est celui qu'utilisaient les indigènes pour se désigner eux-mêmes. Les deux mots sont donc à éliminer, car ils constituent un rappel constant de l'appartenance de certains sujets de l'empereur à un peuple et à une culture différents, ce qui va bien évidemment à l'encontre de la politique d'assimilation du gouvernement de Meiji.

Les aborigènes de Hokkaidō deviennent officiellement des sujets impériaux par la Loi sur l'état civil (kosekihō 戸籍法) de 1871. Ils font alors partie intégrante du «peuple ordinaire » (heimin 平民). Cependant, il est évident qu'ils ne peuvent pas pour autant être désignés par le terme nihonjin 日本人 ( "Japonais ») au même titre que les Japonais, ce qui reviendrait à les mettre sur un pied d'égalité avec ces derniers. Le gouvernement doit donc trouver un terme qui, sans trop les marginaliser, reflète bien leur infériorité par rapport aux Japonais de souche. Ce terme, fabriqué sur mesure, est kyūdojin 旧土人, qui signifie littéralement " ancien indigène $»^{55}$. Or, si le gouvernement japonais cherche véritablement une désignation qui pourrait faciliter l'assimilation, kyūdojin n'est peutêtre pas forcément bien choisi. En effet, il maintient les populations concernées dans le passé et rend flou leur avenir au sein d'un peuple d'avec lequel il marque une séparation très nette. Utilisé dans les documents officiels à partir de 1878, son emploi se raréfie après la fin de l'ère Meiji. Il cesse d'être employé en 1937. En ce sens kyūdojin nous semble être le produit d'un temps où le pays ne se pensait pas encore comme une nation homogène, mais pouvait se concevoir comme un ensemble d'éléments disparates ${ }^{56}$.

51 Le terme générique trouvé, les Aïnous bénéficiant d'un statut propre en tant que peuple, il faut que l'assimilation puisse s'opérer au niveau de l'individu. Il s'agit d'attribuer à chacun une identité propre, passant par l'acquisition d'un nom de famille et d'un nom personnel enregistrés auprès de l'état civil, qui permettent de soumettre nominalement les Aïnous aux nombreuses lois dont ils font l'objet.

Reste le problème de la retranscription à l'aide des caractères japonais de noms personnels incompréhensibles pour une oreille japonaise, qui pâtit forcément de la pauvreté phonétique des katakana et des hiragana, et l'absence de noms de famille. Pour pallier cette dernière, la difficulté est grande, comme dans bien des régions du Japon d'ailleurs, et le mode opératoire choisi par les fonctionnaires n'est pas partout le même : dans le district de Hakodate, par exemple, le nom personnel du chef de famille est repris tel quel, ou sous forme simplifiée, et retranscrit en kanji, de telle sorte que le mot japonais conserve une sonorité proche ${ }^{57}$. Dans le district de Sapporo, tout le village reçoit le même nom de famille, sans souci des liens de parenté ${ }^{58}$. Kayano Shigeru, qui rapporte ce que lui a raconté son grand-père, évoque la venue d'administrateurs ivrognes et incapables dans son village de Nibutani, et leur décision, dans l'urgence le 
jour de leur départ, de n'attribuer que trois noms de famille pour toute la région, sans se soucier des liens de parenté ${ }^{59}$.

Les données des sondages effectués à Hokkaidō pour établir l'identité des Aïnous sont donc inexactes du fait de la déformation des noms personnels et de la distribution au hasard et par des administrateurs peu scrupuleux de noms de famille japonais. Ils permettent toutefois de faire un grand pas sur la voie de la japonisation des populations qu'ils touchent. En effet, pour les générations suivantes qui possèderont un nom de famille et même un nom personnel usuel japonais, l'usage de ces derniers s'imposera $^{60}$. L'interdiction de certaines pratiques importantes accentue encore, sous couvert d'intégration, l'acculturation des Aïnous.

\section{Les interdictions culturelles : rendre les nouveaux Japonais présentables}

54 Si durant les toutes premières années de la Mission, l'assimilation n'est pas une priorité, des mesures visent rapidement à la disparition des «mauvaises habitudes » (rōshū 唒習). Ainsi, les coutumes qui doivent être interdites au plus vite sont celles qui concernent l'aspect physique des Aïnous. En effet, dans l'optique de leur intégration à la population japonaise, il faut éliminer les signes visibles de l'appartenance à un autre peuple et à une autre culture et éviter que ces signes puissent devenir un signe de protestation face à l'autorité japonaise. D'après ce que l'on voit sur les représentations picturales de l'époque d'Edo représentant des Aïnous, ce qui frappait le plus les Japonais était les barbes très fournies et les boucles d'oreille portées par les hommes, ainsi que les tatouages des femmes ${ }^{61}$. Ces deux pratiques sont interdites le 8 octobre 1871, il est vrai, avec d'autres pratiques ayant plus concrètement trait à la " sécurité ", telle que celle d'incendier les maisons (voir plus bas), ou celle touchant à l'armement des chasseurs-collecteurs que sont les Aïnous ${ }^{62}$.

Le fait de devoir mettre fin à des coutumes ancestrales si importantes remplit les Aïnous d'appréhension. Ils se trouvent pris entre leur crainte des représailles divines et leur peur des autorités japonaises ${ }^{63}$. Celle-ci est d'ailleurs rapportée par une voyageuse anglaise, Isabella Bird, dans un ouvrage qui raconte son périple «dans les contrées reculées du Japon » :

Ils [les Aïnous] ressentent à l'égard du gouvernement japonais une peur étrange moi-même je trouve cette peur ridicule. Monsieur Siebold (N.B. membre du personnel de légation austro-hongroise basée à Tōkyō) pense que c'est parce que les administrateurs les menacent et les maltraitent. [...] Ils [les Aïnous] étaient très tristes de la récente interdiction du gouvernement concernant les tatouages, et ils étaient également embarrassés. Les dieux seraient mécontents, et les femmes non tatouées ne pourraient pas se marier, disaient-ils.

彼らは日本政府に対して奇妙な恐怖一私にはばかばかしい恐怖と思われるのだ がーを抱いている。役人たちが彼らを脅迫し酷い目にあわせているからだ、と シーボルト氏（注・東京在柱のオーストリア公使館員）は考えている。[...] 彼 らは最近日本政府が入机墨を禁止したのをたいそう悲しみ、また困惑してい る。神々は怒るだろうし、入れ墨をしなければ女は結婚できないのだ、という ${ }^{64}$ 。

Par ailleurs, la crainte, engendrée cette fois par les représailles divines et par l'impossibilité des femmes non tatouées de se marier, est également perceptible dans ce même ouvrage. Les conséquences de ces interdictions sont importantes. L'institution 
même du mariage traditionnel aïnou serait mise en péril, la continuité des générations mises à mal.

La seconde pratique culturelle à laquelle s'attaque la Mission concerne les rites funéraires: interdiction est faite, en 1871 également, de brûler la maison d'une personne après sa mort (rite appelé chise gomori). Dans l'ensemble, les Aïnous avaient peur des esprits des morts, ces » ombres qui effraient les hommes " (Hito no osoreru kage 人の怖机る影). Pour cette raison, ils n'osaient pas non plus s'approcher des tombes. Dans cette même optique, il fallait empêcher que les esprits des défunts ne reviennent, car ils apporteraient la maladie, assècheraient les sources, et feraient disparaitre gibier et poissons ${ }^{65}$. C'est cette croyance qui était à l'origine du chise gomori, cérémonie au cours de laquelle on brûlait la maison (chise) où le défunt était mort, pour ne pas que son esprit puisse revenir tourmenter les vivants ${ }^{66}$.

On peut se demander quelle était la motivation de l'interdiction précoce de cette pratique. Était-ce dû à la peur de manifestations revendicatrices, galvanisées par la douleur du deuil ? Ce rite funéraire impliquant un groupe d'Aïnous attristés et choqués, munis de torches, allant brûler la maison d'un défunt (peut-être suite à des mauvais traitements de la part des Japonais d'ailleurs) risquait-il de dégénérer en révolte contre l'autorité locale japonaise?

Entre 1869 et 1872, la Mission s'attaque donc aux coutumes qui pourraient lui être les plus nocives et dangereuses, c'est-à-dire celles qui pourraient être à l'origine d'actes de résistance ou constituer des signes trop visibles de différenciation. En parallèle, elle effectue sa première tentative d'éducation ciblée en vue de l'assimilation des populations indigènes.

\section{L'éducation, outil de l'assimilation : l'École temporaire de la Mission au défrichement}

60 La Mission a compris très tôt qu'un des vecteurs les plus efficaces de l'assimilation des Aïnous serait une éducation ciblée et spécifique qui ferait des enfants des futures générations loyaux sujets du souverain.

Dès 1872, trente-cinq individus sont séparés de leurs familles et envoyés à Tōkyō, à l'École temporaire de la Mission au défrichement (Kaitakushi karigakkō 開拓使仮学校). Vingt-six garçons et neuf filles, âgés de treize à trente-huit ans, et issus de Yoichi, Otaru, Takashima, Ishikari et Sapporo, participent à cette expérience éducative ${ }^{67}$.

Les matières qui leur sont enseignées sont la lecture, l'écriture, les idéogrammes de base, le jardinage, l'agriculture et l'élevage. Ce choix n'est évidemment pas anodin. L'enseignement des bases de la langue japonaise devait à la fois permettre une meilleure communication avec les Japonais, mais aussi contribuer au déclin de leur langue maternelle aïnoue. Cependant, ce japonais « de base » ne leur permet pas d'être considérés comme les égaux des Japonais, ni d'accéder à des professions élevées. D'ailleurs, et c'est ce que l'on peut voir d'après les autres matières enseignées, les autorités japonaises ont déjà tracé leur avenir professionnel : les Aïnous deviendront agriculteurs, et de ce fait, acteurs de l'exploitation de Hokkaidō. C'était d'ailleurs le projet de la Mission au défrichement dès son départ, et l'une des motivations de l'abolition du système des basho. Cela restera un moteur constant de son action ${ }^{68}$. 
63 Après seulement quelques années, l'expérience de l'École temporaire de la Mission au défrichement se solde par un échec. Séparés de leur famille et de leur environnement, les sujets de l'expérience fuguent ou tombent malades : au bout de trois ans, tous sont rentrés au pays. Forte de cette expérience, la Mission au défrichement ouvrira en 1877 des écoles spéciales pour enfants, les « écoles pour anciens indigènes » (kyūdojin gakkō 旧土人学校), sur le territoire de Hokkaidō, et l'éducation redeviendra un des axes prioritaires de l'assimilation.

64 Avec la restauration de la monarchie impériale en 1868, le Japon s'efforce de mettre en place les bases de la construction d'un État-nation économiquement viable, capable de rivaliser avec les grandes puissances ouest-européennes et américaine. Après une longue période de morcellement du territoire et de sa population en fiefs, le Japon de la monarchie de Meiji cherche à unifier le pays et à créer chez ses sujets un sentiment d'appartenance nationale, sous l'égide du souverain. Sa souveraineté s'exerce à travers de nouvelles institutions politiques et administratives modernes. L'annexion de l'île d'Ezo, qui suit de très près l'avènement de Meiji, constitue une rupture dans les rapports de domination du septentrion japonais qui fonctionne comme une sorte de préambule à la construction de l'État-nation japonais, en permettant un renforcement de l'État à travers une expansion territoriale et un développement économique et stratégique.

65 L'annexion va permettre au gouvernement japonais de tester l'établissement sur le territoire nouvellement acquis d'une autorité propre et relativement indépendante, concrétisée par l'instance administrative spécifique qu'est la Mission au Défrichement. Celle-ci s'appuie sur des conseillers américains pour élaborer une politique coloniale qui s'articule autour de trois grandes lignes de force: la prise de possession administrative de l'île; l'organisation du défrichement en vue d'une exploitation économique du territoire; l'instauration de mesures d'assimilation concernant les indigènes. La politique d'assimilation les intègre administrativement en leur conférant un nouveau statut - celui d'" ancien indigène »- et prévoit leur "japonisation » progressive : instauration forcée de patronymes japonisés; interdictions des pratiques culturelles différenciant le plus nettement autochtones et Japonais ; éducation passant par la langue japonaise, et tournée vers l'agriculture.

66 Les mesures - administratives, économiques et assimilatrices - de l'annexion, prises entre 1869 et 1872, constituent une première étape cruciale à l'intégration de l'île à l'espace japonais.

67 Fort de cette première expérience d'annexion, le Japon va amorcer celle du royaume des Ryūkyū, en 1872. Cette coïncidence dans les dates et le décalage temporel entre ces deux annexions nous poussent à croire que la mise en place de celle de l'île d'Ezo, entre 1868 et 1872, peut avoir servi de test au processus d'intégration territorial japonais.

68 En 1872, Hokkaidō est donc un territoire annexé en cours d'intégration, tout comme la population aïnoue est en cours d'assimilation. Si les premières mesures administratives et institutionnelles ont pavé la voie à cette intégration, celle-ci prendra encore de nombreuses décennies. 


\section{NOTES}

1. D'après les cartes européennes de l'époque en possession de Toyotomi Hideyoshi 豊臣秀吉 (1537-1598), responsable de l'invasion de la péninsule coréenne de l'invasion de la péninsule entre 1592 et 1598), à l'instar de celle de Matteo Ricci, l'île d'Ezo aurait été reliée au continent et à la Tartarie (Orankai 兀良哈). Sur l'importance des considérations cartographiques dans la domination de l'île d'Ezo, voir Akizuki Toshiyuki 秋月俊幸, Nihon hokuhen no tanken to chizu no rekishi 日本北辺の探検と地困の歴史 (L'Histoire de la cartographie et de l'exploration du Nord), Hokkaidō daigaku Toshohankōkai 北海道大学図書刊行会, Sapporo, 1999, 405 p. et Kamiya Nobuyuki, "Japanese control of Ezochi and the Role of Northern Koryō", Acta Asiatica, no. 67, 1994, pp. 49-68.

2. Du nom d'une route commerciale reliant le Japon, la Chine et la Russie via les régions longeant le cours de l'Amour.

3. Kokudaka 石高: système de calcul du revenu des terres d'un fief en koku (l'équivalent de cent quatre-vingts litres) de riz en fonction de sa taille et de la qualité de la terre. Ce calcul en koku sert ensuite de base au calcul du revenu des seigneurs et de son imposition.

4. Outre l'aménagement du système de kokudaka évoqué plus haut, les seigneurs (daimyō) de Matsumae bénéficient en effet d'un assouplissement du système de sankinkōtai 参勤交代 (résidence alternée des seigneurs à la capitale et dans leur fief), et d'une liberté de mouvement et commerciale dont l'extension est justifiée par l'éloignement géographique.

5. À ce sujet, voir Inagaki Reiko 稲垣令子, « Kinsei Ezochi ni okeru girei shihai no tokushitsu: uimamu, omusha no hensen o tooshite» 近世蝦夷地における儀礼支配の特質 ウイマム・オム シャの変遷を通して (Les particularités de l'étiquette de la domination : les évolutions dans les cérémonies de uimam et d'omusha), Minshū seikatsu to shisō 民衆生活と思想 (Mode de vie et Mode de pensée du peuple), Minshūshi Kenkyūkai 民衆史研究会, 1985, 253 p., p. 111-130, ou 《Ainu minzoku ni taisuru girei shihai: uimamu, omusha ni tsuite»アイヌ民族に対する儀礼支配〈ウ イマム〉・〈オムシャ〉について (La Domination des Aïnous par le rituel : les uimamu et les omusha), Kita kara no Nihonshi 北からの日本史 (L'Histoire du Japon vue du Nord), Hokkaidō Tōhoku-shi Kenkyukai 北海道東北史研究会, 1988, 346 p., p. 315-321.

6. Voir David L. Howell, Capitalism from Within: Economy, Society and the State in a Japanese Fishery, Berkeley, University of California Press, 1995, 246 p.

7. Parmi ces naufragés, les plus influents furent sans doute Denbei 伝兵衛 en 1696 (il rencontre Pierre le Grand en 1702), Daikokuya Kodayū 大黒屋光太夫 en 1783 (il rencontre Catherine II ; après dix ans passés en Russie, il sert de monnaie d'échange pour l'instauration de relations diplomatiques bilatérales nippo-russes) et Tsudayū 津太夫 (le premier Japonais à avoir effectué une circumnavigation lors de son retour au Japon en 1804). Voir, entre autres, Inoue Yasushi, Rêves de Russie, (traduction par Brigitte Koyama-Richard d'Oroshiyakoku suimutan おろしや国酔夢 譚), Phébus, 1968, $320 \mathrm{p}$.

8. Le Français La Pérouse en 1787, l'Anglais Broughton en 1796 et les Russes Kruzenstern et Golovnine, respectivement en 1804 et 1811. Ce dernier sera d'ailleurs capturé par les Japonais alors qu'il visite l'île de Kounachir, dans les Kouriles, et maintenu en captivité pendant deux ans. 9. Leur chef de file est Matsudaira Sadanobu 松平定信, chef du Conseil entre 1787 et 1793. À ce sujet, voir Herman Ooms, Charismatic Bureaucrat: A Political Biography of Matsudaira Sadanobu 1758-1829, Chicago, University of Chicago Press, 1975, 225 p.

10. Voir Kudō Heisuke 工藤平助 et son « Rapport sur le pays des Ezo rouges [les Russes]» (Akaezo fūsetsukō 赤蝦夷風説考), Hayashi Shihei 林子平 et ses deux ouvrages Sankoku tsūranzusetsu 三国 通覧困説 (Aperçu général des Trois Royaumes) et Kaikoku heidan 海国兵論 (De la défense des pays maritimes), ainsi qu'Aizawa Yasushi 会沢安(également connu comme Aizawa Seishisai 会沢正志 
斎) auteur en 1801 de Chishima Ibun 千島異聞 (Contes étranges de Chishima), consultables dans Terasawa Hajime 寺沢一 (dir.), Hoppō mikōkai kobunsho shūsei 北方未公開古文書集成 (Recueil de textes anciens non accessibles au grand public concernant les territoires du Nord), 10 volumes, Sōbunsha 叢文社. Voir également Annick Horiuchi, « Le Kaikoku Heidan de Hayashi Shihei », Ebisu $\mathrm{n}^{\circ} 38$, Automne-Hiver 2007, p. 83-101.

11. Augustin Berque, "La Chaîne culturelle d'une colonisation : les paysans à Hokkaidō ", Annales. Économies, Sociétés, Civilisations, année 1974, volume 29, nº 6, p. 1425-1449, p. 1428.

12. Insurrection de Kunashiri-Menashi (ou Soulèvements Ezo de l'ère Kansei, Kansei Ezo sōdō 寛政 蝦夷騷動) : révolte aïnoue dans les Kouriles du Sud. Plus de soixante-dix colons sont tués, c'est-àdire la quasi-totalité de la population venue de Honshū dans la région. En représailles, les chefs aïnous à la tête de l'insurrection sont exécutés, et ceux restés "loyaux " à Matsumae sont récompensés. Ces derniers sont immortalisés, en une peinture sur soie de 1791, par le peintre Kakizaki Hakyō 蠣崎波響 (1764-1826), dans une série intitulée Ishū retsuzō 夷酋列像 (Figures des chefs barbares). Sur ce sujet, voir Tahara Kaori, «Réflexions sur une série de peintures sur soie de Kakizaki Hakyō représentant des chefs aïnous ", Japon Pluriel 5, Actes du cinquième colloque de la Société française des Études japonaises, Arles, Éditions Philippe Picquier, 2004, 469 p., p. 199-210.

13. Rodolfo Stavenhagen, The Ethnic Question: Conflicts, Development and Human Rights, Tōkyō, United Nations University Press, 1991, 185 p., p. 3.

14. Ce concept décrit l'établissement progressif d'une société sur le territoire d'une autre dans un but d'expansion territoriale et de développement économique, et ce au cours d'un long processus durant lequel la "société supplantante " émet des revendications territoriales supérieures à celles de la société indigène, mais surtout supérieures à celles d'une autre société qui pourrait la remettre en question. David Day, Conquest - How Societies Overwhelm Each Other other, New York, Oxford University Press, 2008, 288 p., p. 6.

15. Pierre-François Souyri, « Une forme originale de domination coloniale? Les Japonais et le Hokkaidō avant l'époque Meiji », De Russie et d'ailleurs: Feux croisés sur l'histoire: pour Marc Ferro, Institut d'études slaves, 1995, 607 p., p. 373-386, p. 374.

16. Le Traité de Kanagawa 神奈川条約 (Kanagawa jōyaku), ou Traité d'amitié nippo-américain 日 米親和条約 (Nichibei Shinwa jōyaku), est signé le 31 mars 1854 entre le Japon et les États-Unis. Qualifié par les Japonais de premier des «traités inégaux» fubyōdō jōyaku 不平等条約 que le Japon (et avant lui la Chine) est obligé de signer avec les autres puissances européennes. Il est considéré comme le premier traité mettant fin à la politique dite du Sakoku, c'est-à-dire du verrouillage maritime partiel du Japon, conséquence des interdictions maritimes mises en place dans les années 1630. Il stipule principalement que les ports de Shimoda et Hakodate sont ouverts aux navires américains, qui sont en droit d'y obtenir des vivres et du combustible, ainsi que d'y pratiquer des échanges de denrées, et l'installation d'un consulat à Shimoda.

17. Voir Thierry Mormanne, «Le Problème des Kouriles : pour un retour à Saint-Pétersbourg ", Cipango, 1992-1, p. 59-89.

18. Ogasawara Nobuyuki 小笠原信之, Ainu kingendaishi yomihon アイ又近現代史読み本 (Histoire contemporaine et actuelle des Aïnous), Ryokufu shuppan 緑風出版, 2001 (2 édition), 278 p., p. 12.

19. Shin.ya Gyō 新谷行, Ainu minzoku to nihon tennōsei kokka アイヌ民族と日本天皇制国家 (Le Peuple aïnou et l'État monarchique japonais), San.ichi shobō 三一書房, 1978, 398 p., p. 334.

20. Après la chute d'Edo aux mains des troupes monarchistes, le commandant de la marine shogunale, Enomoto Takeaki, part avec sa flotte (huit navires de guerre et deux mille hommes) vers le nord, dans l'espoir de lancer une contre-attaque. Arrivés à Hokkaidō, ils fondent la République d'Ezo (Ezo kyōwakoku 蝦夷共和国) le 25 décembre 1868, avec à sa tête Enomoto comme président, et défendue par la forteresse Goryōkaku. À la suite de la bataille navale de Hakodate, les troupes impériales encerclent la forteresse et Enomoto se rend le 17 mai 1869. 
21. Par l'ordonnance 734 intitulée « Rebaptiser Ezochi en Hokkaidō, le diviser en onze régions et fixer le nom des régions et des districts» (Ezochi o Hokkaidō to kaishōshi jūichi kuni ni bunkatsu kokumei gunmei o sadamu 蝦夷地习北海道卜改稱十一ヶ國二分割國名郡名习定ム).

22. Matsuura Takeshirō (1818-1888): ce géographe a déjà participé à trois expéditions d'exploration sur l'île d'Ezo et en a dressé des cartes en 1845. Il a par ailleurs été sous-directeur du siège gouvernemental de Hakodate (Hakodate-fu 箱館府), l'organe administratif régissant l'île d'Ezo entre 1868 et juillet 1869 .

23. Hayashi Yoshishige 林善茂, «Kaitakushi no zaisei kibo » 開拓使の財政規模 (Champ d'action et politique financière de la Mission au défrichement), Hokkaidō daigaku nōkei ronsō 北海道農經論 叢 (Revue universitaire d'économie et d'agriculture de l'Université de Hokkaidō), no. 20, Sapporo, Hokkaidō Daigaku shuppansha 北海道大学出版社, novembre 1963, p. 1-12, p. 2.

24. Entre février 1870 et août 1871, la Mission au défrichement de Sakhaline (Karafuto kaitakushi 樺太開拓使) voit éphémèrement le jour. La signature du Traité de Saint-Pétersbourg, ou Traité d'échange entre les îles Kouriles et Sakhaline (Chishima Karafuto kōkan jōyaku 千島樺太交換条約), signé entre le Japon et la Russie en mai 1875 met fin à toute politique de défrichement japonaise de l'île.

25. En parallèle, des antennes régionales sont installées - durablement cette fois - à Tōkyō, Hakodate, Nemuro, Sōya, Uraga et Sakhaline.

26. Avant Meiji, les Aïnous étaient désignés par de nombreuses appellations, dont Ainu, Ezo, dojin 土人 (indigène), ijin 異人/夷人 (personne différente/barbare), etc.

27. Ce terme, hérité du modèle de classification géographique antique, a le sens de « circuit ».

28. Le texte original peut être consulté sur internet.

29. Lors d'une expédition pour enquêter sur la possibilité de construire des routes vers la rivière Teshio, en 1857, Matsuura avait été accueilli par la famille d'un vieil Aïnou appelé Aetomo. Celuici lui avait indiqué que Kaina venait de l'aïnou kai, qui signifie « homme né dans ce pays ", suivi du suffixe honorifique na. Voir Ogasawara Nobuyuki, Ainu kingendaishi yomihon, op. cit., p. 10.

30. Takenouchi Sukune 武内宿禰 : personnage légendaire qui aurait servi plusieurs souverains entre le $\mathrm{I}^{\mathrm{er}}$ et le $\mathrm{IV}^{\mathrm{e}}$ siècle en tant que général et aurait combattu les Ebisu, peuple vivant au nord de Honshū et à Hokkaidō, assimilé à l'époque moderne et contemporaine aux Aïnous.

31. Shin.ya Gyō, Ainu minzoku to nihon tennōsei kokka, op. cit., p. 334.

32. Parallèlement, de nombreux lieux et îles périphériques vont être dotés de nouveaux noms, parfois issus des appellations d'origine, le plus souvent altérées. Voir par exemple Hamada Ryūji 浜田龍司, Kaitaku：Hokkaidō no rekishi 開拓一北海道の歴史 (Défrichement: l'Histoire de Hokkaidō), Mainichi shinbunsha 毎日新聞社, 1974, 245 p., p. 49.

33. Hayashi Yoshishige, « Kaitakushi no zaisei kibo », op. cit., p. 2.

34. Uehara Tetsusaburō 上原轍三郎, «Hokkaidō kaitaku kyokushoki ni okeru tochi seido » 北海 道開拓極初期に於ける土地制度 (Le Système terrien des toutes premières années de l'exploitation de Hokkaidō), Hōkeikai ronsō 法經會論叢 (Collection d'essais en Droit et Économie), $\mathrm{n}^{\circ}$ 6, Sapporo, Hokkaidō Daigaku shuppansha, mars 1938, p. 1-36, p. 7.

35. Torisu Keiichi 鳥巣京一, «Meiji shonen Kyūshū shohan to Hokkaidō kaitaku » 明治初年九州 諸藩と北海道開拓 (Les fiefs de Kyūshū et le défrichement de Hokkaidō dans les premières années de l'ère Meiji), Keizaigaku kenkyū 経済学研究 (Études en sciences économiques), n 69 (3/4), Kyūshū Daigaku Shuppan 九州大学出版, 2003, p. 16.

36. Ogasawara Nobuyuki, Ainu kingendaishi yomihon, op. cit., p. 37.

37. Kikuchi Yoshiki 菊池芳樹, «Kokunai jinkō idō no kenkyū - Hokkaidō no jinkō hendō 1869-1925 » 国内人口移動の研究 北海道の人口变動 1869 1925 (Étude des mouvements de populations internes - Évolutions de la population de Hokkaidō 1869-1925), Shakaigaku Kenkyūka kiyō 社会学研究科紀要 (Revue universitaire du département d'études sociologiques), $n^{\circ}$ 21, Keiō Daigaku Shuppansha 慶応大学出版社, 1981, p. 21-34, p. 22. Par ailleurs, on considère 
qu'à partir de 1750 la masse d'habitants en provenance de Honshū est déjà supérieure à celle des Aïnous. Référence en ligne.

38. Tsubo 坪: unité de mesure de surface équivalente à deux tatami, soit environ 3,35 mètres carrés.

39. Kikuchi Yoshiki, « Kokunai jinkō idō no kenkyū - Hokkaidō no jinkō hendō 1869-1925 », op.cit., p. 22.

40. Takakura Shin.ichirō 高倉新一郎, «Dantai imin no seichō » 團體移民の成長 (Croissance de l'immigration de masse), Hōkeikai ronsō 法經會論叢 (Collection d'essais en droit et économie), $\mathrm{n}^{\circ}$ 11, Hokkaidō daigaku shuppansha, février 1945, p. 173-186, p. 173.

41. Deux cent cinquante personnes arrivent en avril 1870, rejointes par soixante-douze en août. Ils sont sept cent quatre-vingt-huit personnes l'année suivante. En 1881, après neuf vagues d'envoi, un total de deux mille six cent quatre-vingt-une personnes arrivent dans le sillon de la Maison Date.

42. Extrait du roman Gendaishi no naka no hitori 現代史の中の一人 (Seul dans l'histoire de notre temps), cité dans Kaiho Mineo 海保嶺夫, Kinsei no Hokkaidō 近世の北海道 (Hokkaidō à l'époque moderne), Kyōiku shakaishi shinsho 教育社会史新書, Nihonshi 99 日本史 99, Kyōikusha 教育社, 1979, 220 p., p. 12.

43. Idem.

44. Sorti en 2005 au Japon, le film a été récompensé par un Japanese Academy Award en 2006.

45. Cette fête a lieu chaque année en novembre et, comme son nom l'indique elle commémore le repas d'action de grâce des colons anglais après avoir survécu au premier hiver en Amérique, grâce à l'aide inattendue d'un Indien du nom de Squanto (Tisquantum), de la tribu des Patuxet, qui aida ces colons à survivre.

46. John A. Harrison, "The Capron Mission and the Colonization of Hokkaidō", Agricultural History, volume 25, no. 3, Juillet 1951, pp. 135-142, p. 136.

47. Kuroda Kiyotaka (1840-1900) : homme politique japonais. En tant que sous-directeur du Kaitakushi, il est à l'origine du Traité de Saint-Pétersbourg (1875), qui stipule l'échange avec la Russie de l'île de Sakhaline contre l'archipel des Kouriles, et de la création des troupes de soldatspaysans, les tondenhei (voir infra). En 1877, il est nommé ministre de l'Agriculture, puis il devient Premier ministre en 1888 , et ce jusqu'en 1889. Il devient alors ministre des Communications en 1892 et président du Conseil privé en 1895.

48. Horace Capron (1804-1885) est décoré, en 1884, pour ses travaux à Hokkaidō, de l'Ordre du Soleil Levant (kyokujitsu shō 旭日章), par Meiji. Pour plus de détails sur cet homme, voir sa correspondance avec la Mission (Reports and Official Letters to the Kaitakushi, by Horace Capron and his foreign advisors and assistants).

49. Shin.ya Gyō, op. cit., p. 333.

50. Ainsi que par les lois suivantes : la Loi de Distribution des Actes de propriété terrienne de Hokkaidō de 1877 (Hokkaidō chiken hakkō jōrei 北海道地券発行条例), la Loi de Régulation du transfert des terres gouvernementales à la propriété privée de 1886 (Hokkaidō tochi harai kudari kisoku 北海道土地迅下規 則) et la Loi sur les terres gouvernementales non défrichées de Hokkaidō de 1897 (Hokkaidō kokuyū mikaichi shobunhō 北海道国有未開地処分法).

51. Nelcya Delanoë, L'entaille rouge : des terres indiennes à la démocratie américaine (1776-1996), Paris, Albin Michel, coll. « Terre indienne », 1996, p. 74.

52. Kikuchi Yoshiki, « Kokunai jinkō idō no kenkyū - Hokkaidō no jinkō hendō 1869-1925 », op. cit., p. 22.

53. Kayano Shigeru 萱野茂 (1926-2006), spécialiste de l'histoire et de la culture aïnoues et premier Aïnou à avoir siégé à la Diète (1994-1998), évoque dans ses mémoires (en anglais sous le titre Our Land was a Forest: An Ainu Memoir, Westview Press, 1994, 192 p.) l'histoire de son grandpère Tokkaramu, employé de force dans un basho. Les garçons de son village étaient emmenés à partir de douze ans à trois cents kilomètres de leur lieu d'origine pour travailler dans l'industrie 
piscicole. Il semblerait en effet qu'en 1858, dans la région de la rivière Saru, d'où est originaire la famille de Kayano, 82,3 \% des hommes de onze à cinquante ans et 35,7 \% des femmes du même âge sont envoyés dans les basho d'Ishikari, Otaru et Astukeshi. Cf. Ogawa Masato 小川正人, «"Ainu gakkō" no setchi to "Hokkaidō kyūdojin hogohō", "Kyūdojin jidōkyō" no seiritsu »『ア イヌ学校』の設置と「北海道旧土人保護法」・「旧土人児童教」の成立 (L'implantation des «écoles aïnoues » et la mise en place de la Loi de Protection des Anciens Indigènes et de l'Éducation des jeunes anciens indigènes), Hokkaidō Daigaku kyōiku gakubu kiyō 北海道大学教育学 部紀要 (Revue universitaire du Département d'Enseignement de l'Université de Hokkaidō), n 55, mars 1991, p. 257-325, p. 266.

54. John A. Harrison, "The Capron Mission and the Colonization of Hokkaidō", op. cit., p. 138.

55. Ce mot est composé de l'idéogramme kyū 旧 et du composé dojin 土人, signifiant respectivement " ex -», " ancien ", et « indigène », " autochtone », " aborigène ». Je choisis de le traduire par "ancien indigène » car en français, le terme "aborigène » est souvent lié à la population indigène de l'Australie, et que le terme "autochtone " ne reflète, à notre sens, pas assez la volonté japonaise d'asseoir, en utilisant ce terme, sa supériorité économique, politique, technique et civilisationnelle.

56. Sur ce changement de paradigme, A. Nanta, "Altérité aïnoue dans le Japon moderne (1880-1900) ", Annales. Histoire, Sciences Sociales, 2006/1, 61 e année, p. 247-273.

57. Ogasawara Nobuyuki, Ainu kingendaishi yomihon, op. cit., p. 40.

58. Idem.

59. Kayano Shigeru, Our Land was a Forest: An Ainu Memoir, op. cit., p. 57-58.

60. Avant leur assimilation à l'état civil japonais, les Aïnous, qui ignoraient l'écriture, ne possédaient pas de patronymes, mais un double système pour établir des généalogies patrilinéaires et matrilinéaires. Les ekashi shiroshi («symboles de l'homme âgé »), transmis de génération en génération et gardés secrets à l'intérieur d'une famille permettaient d'établir la lignée patrilinéaire. Chez les femmes, les motifs tressés des upsor kut (« ceinturons poitrinaires ») servaient à marquer la lignée matrilinéaire. Ils étaient également transmis de génération en génération. Par ailleurs, voir Endō Masatoshi 遠藤匡俊, «Jūkyū seiki no ainu shakai ni okeru wameika no tenkai katei»19世紀のアイヌ社会における和名化の展開過程 (La vague de japonisation des patronymes japonais dans la société aïnoue au XIX siècle), Chiri zasshi 地理雑誌, volume 113, $\mathrm{n}^{\circ}$ 3, 2004, p. 421-424.

61. Rappelons que dans la culture japonaise, le tatouage est associé à la pègre et une marque de souillure. Pour les Aïnous, au contraire, le tatouage des femmes autour de la bouche marquait l'accession à l'âge adulte. Complété au mariage, il aurait symbolisé le fait que toute parole qui sortirait désormais de la bouche de la femme appartiendrait à son mari. Inspiré par la sœur cadette du Dieu créateur, le tatouage était porté en signe de vénération religieuse, ainsi que pour se prévenir de l'entrée de mauvais esprits dans le corps ; Chikappu Mieko チカップ美恵子, Ainu moshiri no kazeアイヌ・モシリの風 (Vents du pays aïnou), Nihon hōsō shuppan kyōkai 日本放送 出版会, 2001, 254 p., p. 76.

62. Lors du contrôle provisoire de l'île d'Ezo par le shogunat entre 1799 et 1821, une «politique d'amélioration des mœurs" (kaizoku seisaku 改俗政策) avait déjà été amorcée. Dans ce cadre avaient été interdits la fête rituelle de sacrifice de l'ours iomante et les tatouages. Ces mesures sont abolies en 1821, à la fin de la gestion shogunale de l'île, en même temps que l'usage de la langue japonaise est à nouveau interdit aux Aïnous.

63. Quiconque ne se plie pas à cette loi encourt en effet une sévère peine de prison; Ogasawara Nobuyuki, Ainu kingendaishi yomihon, op. cit., p. 42.

64. Isabella Bird, Nihon okuchi kikō 日本奥地紀行 (Récits d'un voyage dans les contrées reculées du Japon), no. 36, rapporté à partir de l'édition japonaise par Ogasawara Nobuyuki, Ainu kingendaishi yomihon, op. cit., p. 44. Isabella Bird effectue, à l'âge de quarante-sept ans, un voyage au Japon pour améliorer sa santé. Elle visite pendant trois mois, en 1878, le Tōhoku et Hokkaidō, en 
consignant soigneusement ses impressions dans les lettres qu'elle envoie à sa sœur et à ses amis. Celles-ci seront publiées en anglais en 1880.

65. Arlette et André Leroi-Gourhan, Un Voyage chez les Aïnous : Hokkaidō 1938, Albin Michel, 1989, 156 p., p. 111.

66. Sarashina Genzō 更科源蔵, Ainu to nihonjin アイヌと日本人 (Aïnou et Japonais), NHK Books, 1970, 252 p., p. 133.

67. Ogawa Masato, «"Ainu gakkō” no setchi to "Hokkaidō kyūdojin hogohō”, "Kyūdojin jidōkyō" no seiritsu ", op. cit., p. 271.

68. En 1875, une première série de mesures interdit les pratiques de la chasse et la pêche traditionnelles, et limite la quantité de gibier à la disposition des Aïnous. Ceci les rendra de plus en plus dépendants de l'agriculture. La mise en place de la Loi de protection des anciens indigènes de 1899 consacrera définitivement la politique visant leur assimilation par le travail.

\section{RÉSUMÉS}

Parachevant des siècles de rapports inégaux de nature coloniale, l'annexion de l'île de Hokkaidō par le Japon en 1869 marque une rupture dans la nature, les objectifs et les moyens de la domination japonaise sur l'île. La restauration de Meiji et les troubles intérieurs qui s'ensuivent, la levée des interdictions maritimes isolationnistes, ainsi que la sécurisation de la frontière nord face à la Russie justifient une nouvelle politique coloniale qui s'articule autour de trois grandes lignes de force : la prise de possession administrative et le peuplement de l'île ; l'organisation du défrichement en vue d'une exploitation économique de ses ressources; et l'instauration de mesures d'assimilation concernant les indigènes, les Aïnous. Une instance administrative spécifique est créée pour piloter les opérations : la Mission au Défrichement.

Ces mesures administratives, économiques et assimilatrices, mises en place entre 1869 et 1872, se démarquent profondément des politiques de domination précédentes, et rapprochent le gouvernement de Meiji de l'accomplissement de son objectif principal : construire un État-nation puissant sur les plans économique et militaire, capable de devenir la première grande puissance asiatique.

\section{INDEX}

Thèmes : histoire

Index géographique : Hokkaidō

Mots-clés : Aïnous, assimilation, Mission au défrichement, rapports inégaux

Keywords : Ainu people, Assimilation, Unequal Relations, Hokkaido, Mission of Reclamation, Meiji Period, History

Index chronologique : Meiji (1868-1912) 
AUTEUR

NOÉMI GODEFROY

Centre d'études japonaises, Inalco 